\title{
Power Optimistic with Throughput Improved Adaptive CSMA MAC Protocol Design for Wireless Ad Hoc Network
}

\author{
R. Manikandan, \\ Assistant Professor, \\ Dept.of computer science \& engg., \\ Annamalai University, \\ Chidambaram.
}

\author{
K. Selvakumar, PhD. \\ Associate Professor, \\ Dept.of computer science \& Engg., \\ Annamalai university, \\ Chidambaram.
}

\begin{abstract}
Power conservation and throughput management is a major issue in Mobile Ad Hoc Networks. In the design of wireless ad hoc networks, various techniques are applied to efficiently allocate the scarce resources available for the communication links and the power control and throughput management is not related to any particular layer in the layered design communication protocol design. But most of the power control and throughput management mechanisms are working in MAC layer. An adaptive CSMA medium access control (MAC) protocol is proposed to consider a spatial network model in which nodes are randomly distributed in space, and address the problem of interference, power control, and throughput improvement through CSMA MAC layer design. Power control and throughput improvement is a critical issue to implement Mobile Ad Hoc networks. The proposed method present a novel power control protocol, and improve the aggregate throughput of the network for its possible application in Mobile Ad Hoc networks.
\end{abstract}

Keyword - Ad hoc networks, Poisson point process, MAC protocols, outage probability.

\section{INTRODUCTION}

In the design of wireless ad hoc networks, various techniques are applied to efficiently allocate the scarce resources available for the communication links. Using an appropriate medium access control (MAC) protocol is one such technique. MAC data communication protocol is a sub layer of the data link layer. The sub layer provides addressing and channel access control mechanisms that make it possible for several terminals or network nodes to communicate within a multiple access network that incorporates a shared medium [1]. The channel access control mechanisms provided by the MAC layer are also known as a multiple access protocol. The multiple access protocol may detect or avoid data packet collisions if a packet mode contention based channel access method is used, or reserve resources to establish a logical channel. The ALOHA method is the common multiple access protocols that may be used in packet radio wireless networks; it is limited by the large vulnerability period of a packet. By listening before transmitting, stations try to reduce the vulnerability period to one propagation delay. So the proposed MAC Protocol Layer design using contention based CSMA/CD protocol used in Ethernet networks. This mechanism is only utilized within a network collision domain, for example an Ethernet bus network or a hub-based star topology network. An Ethernet network may be divided into several collision domains, interconnected by bridges and switches [2].
Carrier Sense Multiple Access with Collision Detection (CSMA/CD) is a one of the MAC carrier sensing scheme. A transmitting data station that detects another signal while transmitting a frame, stops transmitting that frame, transmits a jam signal, and then waits for a random time interval before trying to resend the frame. CSMA/CD is modification of pure carrier sense multiple access (CSMA). CSMA/CD is used to improve CSMA performance by terminating transmission as soon as a collision is detected, thus shortening the time required before a retry can be attempted [3]. In this paper proposes a spatial network model in which nodes are randomly distributed in space, and it address the problem of interference through MAC layer design. The CSMA MAC protocol is employed for communication, and the success rate of packet transmissions is investigated.

The rest of this paper is organized as follows. In Section II related work of this paper. In Sections III and IV described Methodology and Simulation Setup. In Section V looks Result and discussions. And Finally Section VI presents the conclusion.

\section{RELATED WORKS}

The performance of the ALOHA and CSMA MAC protocols are analyzed in spatially distributed wireless networks. The main system objective is correct reception of packets, and thus the analysis is performed in terms of outage probability. In our network model, packets belonging to specific transmitters arrive randomly in space and time according to a 3-D Poisson point process, and are then transmitted to their intended destinations using a fully-distributed MAC protocol. A packet transmission is considered successful if the received SINR is above a predefined threshold for the duration of the packet. Accurate bounds on the outage probabilities are derived as a function of the transmitter density, the number of backoffs and retransmissions, and in the case of CSMA, also the sensing threshold. The analytical expressions are validated with simulation results. For continuous-time transmissions, CSMA with receiver sensing (which involves adding a feedback channel to the conventional CSMA protocol) is shown to yield the best performance. Moreover, the sensing threshold of CSMA is optimized. It is shown that introducing sensing for lower densities (i.e., in sparse networks) is not beneficial, while for higher densities (i.e., in dense networks), using an optimized sensing threshold provides significant gain. [4].

A MAC protocol for mobile ad hoc networks that uses power control for the RTS/CTS and DATA frame transmissions in order to improve energy and capacity utilization efficiency. Unlike IEEE 802.11, in our scheme the RTS frames are not sent using the maximum transmission power to silence neighboring nodes, and the CTS frames do not silence all receiving nodes to 
the same degree. In contrast, the transmission power of the RTS frames follows a slow start principle, while the CTS frames, which are sent at maximum transmission power, prevent the neighbouring nodes from transmitting their DATA frames with power more than a computed threshold, while allowing them to transmit at power levels less than that threshold.

This is done by including in the RTS and the CTS frames additional information, such as the power of the transmissions, and the interference tolerance of the nodes. Moreover the DATA frames are sent at the minimum required transmission power increased by a small margin to ensure connectivity with the intended receiver, so as to cause minimal interference to neighbouring nodes and allow for future interference to be added to the receiver of the DATA frames. The power to be used by the transmitter is computed by the recipient of the RTS frame and is included in the CTS frame. It is expected that a network with such a power management scheme would achieve a better throughput performance and more power savings than a network without such a scheme [5]

The performance of unslotted ALOHA and CSMA are analyzed in spatially distributed wireless networks. Users/packets arrive randomly in space and time according to a Poisson process, and are thereby transmitted to their intended destinations using a fully-distributed MAC protocol (either ALOHA or CSMA). An SINR-based model is considered, and a packet transmission is successful if the received SINR is above a threshold value for the duration of the packet. Accurate bounds to the probability of outage, which is a function of the density of transmissions, are developed for both MAC protocols. These bounds are used to evaluate the performances of ALOHA and CSMA, and to gain insight into the design of general MAC protocols for ad hoc networks. Moreover, CSMA with receiver- sensing is proposed to improve the performance of CSMA [6].

Outage probabilities and single-hop throughput are two important performance metrics that have been evaluated for certain specific types of wireless networks. For the performance analysis, a new parameter, the so- called spatial contention, is defined. It measures the slope of the outage probability in an ALOHA network as a function of the transmit probability $p$ at $p$ $=0$. Outage is defined as the event that the signal-tointerference ratio (SIR) is below a certain threshold in a given time slot. It is shown that the spatial contention is sufficient to characterize outage and throughput in large classes of wireless networks, corresponding to different positions on the uncertainty cube. Existing results are placed in this framework, and new ones are derived. Further, interpreting the outage probability as the SIR distribution, the ergodic capacity of unitdistance links is determined and compared to the throughput achievable for fixed (yet optimized) transmission rates [7].

\section{METHODOLOGY}

The modules which are to be implemented in this project are given below.

\subsection{Cooperative Diversity in Wireless Ad Hoc Network}

Cooperative diversity is a novel technique proposed for conveying information in wireless ad hoc networks, where closely located single-antenna network nodes cooperatively transmit and/or receive by forming virtual antenna arrays [8].

\subsection{Implementation Of MAC (CSMA) Protocol}

Due to the poor performance of unslotted ALOHA by introducing channel sensing and the ability to back off from transmissions, the performance of wireless networks was greatly improved. Moreover, several modifications were proposed in order to overcome the inherent hidden and exposed node problems of CSMA. By allowing some kind of communication between the TX and its RX, throughput improvement was achieved. And extended the work to consider point-to-point wireless ad hoc networks and we evaluate the OP performance of CSMA.

In this design, a packet is backed off if the measured or estimated (depending on whether the RX or TX are sensing) SINR is below the sensing threshold, $\beta$ sens, at the beginning of its transmission. Up to a maximum of $M$ times, the packet then waits a random received in error at its $\mathrm{RX}$, the packet is retransmitted. This is repeated $N$ times before the packet is dropped.

\subsection{Outage Probability Of CSMA}

$$
\begin{aligned}
& \text { The total OP of CSMA may be expressed } \\
& \text { as } P_{\text {out }}(\operatorname{csu} A)=P_{b}^{M}+\left(1-P_{b}^{M N}\right) P_{y t 1} P_{y t}^{M}
\end{aligned}
$$

and the density of packets attempting to access the channel is

$$
\lambda_{\operatorname{cgma}}\left(P_{b} P_{y+1} P_{y t}\right)=\lambda\left\lceil\frac{1-P_{b}^{M}}{1-P_{b}}+\left(1-P_{b}^{M}\right) P_{y t 1} \frac{1-P_{y t}^{M}}{1-P_{y t}}\right\rceil
$$

Where Prt 1 is the probability that an activated packet is received erroneously at its first transmission attempt and must be retransmitted, and Prt is the probability of error in the retransmission attempts.

Due to the backoff property of CSMA, and since packets tagged for retransmission do not perform new channel sensing, multiply $\lambda c s m a$ by $(1-P b)$ to find the density of active packets:

$$
\lambda_{\text {active }}\left(P_{b v} P_{y t 1} P_{y t}\right)=\lambda\left(1-P_{b}^{M}+\left(1-P_{b}^{M}\right) P_{y t 1} \frac{1-P_{y t}^{M}}{1-P_{y t}}\right)
$$

\subsubsection{CSMA with Transmitter Sensing}

In the conventional CSMA protocol, which is employed in many of today's network standards, such as IEEE 802.11 and 802.16 , the TX is the backoff decision maker. That is, when a new packet arrives, the TX immediately measures the aggregate interference power. If this is greater than it $\frac{p R-a}{\beta}-\frac{\pi}{\beta}$, backs off, otherwise, it starts transmitting.

$$
\begin{aligned}
& P_{b} \approx \tilde{P}_{b} \\
& \text { is the backoff probability, and is found as the }
\end{aligned}
$$

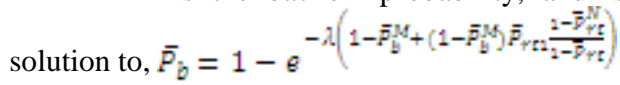

$P_{\gamma t} \otimes \bar{P}_{b}+\left(1-\bar{P}_{b}\right) \bar{P}_{\text {WW }}^{\text {TWinging }}$ is the probability that an activated packet is received erroneously in a retransmission attempt, with $\bar{P}_{\text {during }}$ being the probability that the error occurs at some $t \in$

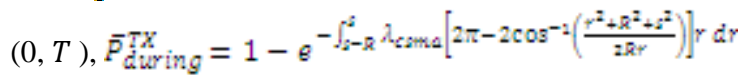

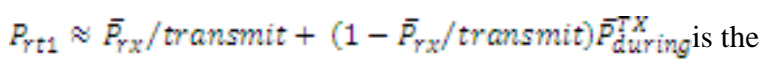
probability that an activated packet is received erroneously at the first transmission attempt, $\tilde{P}_{r x \mid \text { transmit }}$ with being the probability that the RX is in outage upon the packet arrival, given its TX decides to transmit,

$$
\bar{P}_{r x y} \text { tromsmit }=\bar{P}_{b}\left[1-\frac{1}{\pi s^{2}}\left(2 s^{2} \cos ^{-2}\left(\frac{R}{2 s}\right)-R s \sqrt{1-\frac{R^{2}}{4 s^{2}}}\right)\right]
$$




\subsubsection{CSMA with Receiver Sensing}

With the objective of improving the performance of CSMA, introduced a novel protocol, termed CSMARX. In this protocol, the RX senses the channel and subsequently determines whether or not the packet transmission should be initiated. The communication between the TX and RX is assumed to occur over a separate 1 bit control channel, and the delay introduced by the feedback is assumed to be small and insignificant compared to the packet length.

$P_{b} \propto \bar{P}_{b}$ is the backoff probability and

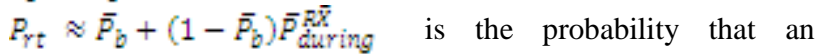
activated packet is received erroneously some time during its transmission and must thus be retransmitted,

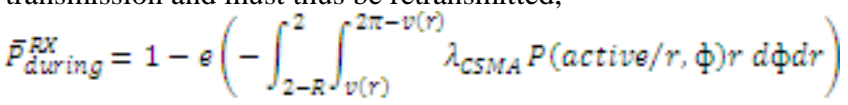

$P\left(\right.$ active $\left./ r_{0} \phi\right)=1-\frac{1}{\pi} \cos ^{-1}\left(\frac{r^{2}+2 R^{2}-s^{2}-2 R r \cos \phi}{\left.2 R_{\sqrt{r^{2}+R^{2}-2 R r \cos \phi}}^{\sqrt{2}}\right)}\right.$

$$
\mathbb{E}(r)=\cos ^{-1}\left(\frac{r^{2}+2 R s-s^{2}}{2 R r}\right)
$$

\subsection{Performance Analysis}

Extensive simulation results of the proposed CSMAMAC protocol along with ALOHA. In the simulations, the source nodes always have data packets to send and the following performance metrics are evaluated.

$$
\begin{array}{ll}
\text { - } & \text { Throughput } \\
\text { - } \quad \text { Energy efficiency }
\end{array}
$$

Finally, CSMA-MAC can significantly improve the performance of throughput and energy efficiency compared to ALOHA.

\subsection{Data Flow Diagram}

The data flow diagram of the ad-hoc Node model and the adaptive CSMA MAC module is given below.

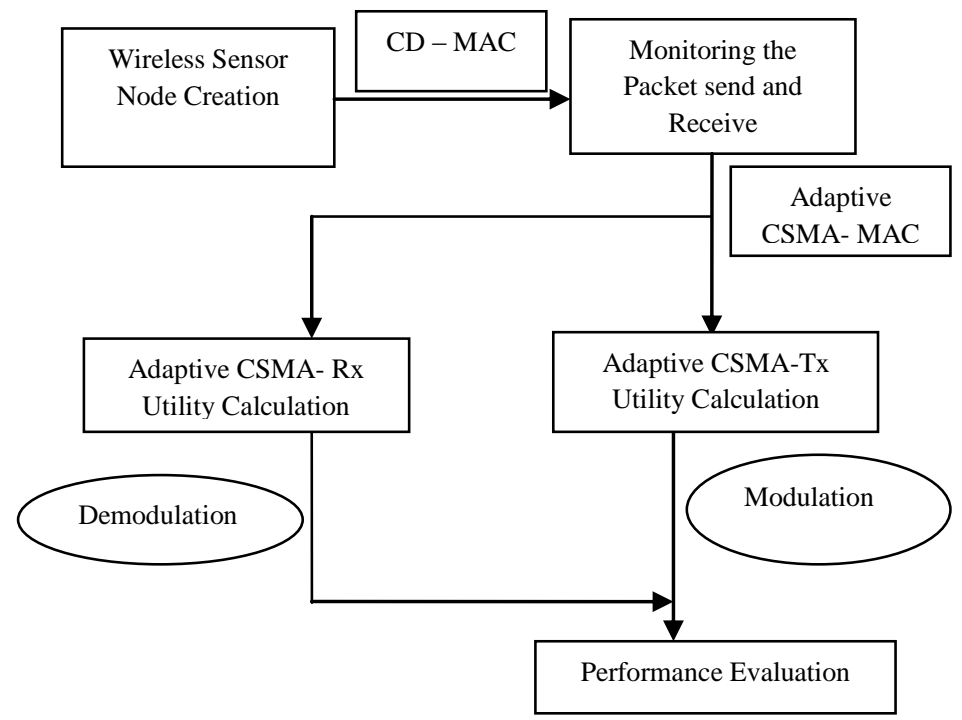

\section{SIMULATION SETUP}

A network simulator is a software program that imitates the working of a computer network. The simulator can be used for traffic modeling of telecommunication networks, protocol modeling, modeling queuing networks, modeling multiprocessors and other distributed hardware systems, validating hardware architectures, evaluating performance aspects of complex software systems. NS2 needs the fixed parameter setup, they given below

Table 1: Simulation Parameters

\begin{tabular}{|l|l|}
\hline \multicolumn{1}{|c|}{ PARAMETER } & \multicolumn{1}{c|}{ VALUES } \\
\hline Mobile nodes & 50 Nodes over 300m x 1500m \\
\hline Reflection model & Two-ray ground reflection model \\
\hline Data rate & 2Mbps \\
\hline $\begin{array}{l}\text { Environment noise } \\
\text { level }\end{array}$ & -83 or $-90 \mathrm{dBm}$ \\
\hline Traffic model & UDP based CBR traffic \\
\hline Routing protocol & $\begin{array}{l}\text { Ad hoc On demand Distance Vector } \\
\text { (AODV) }\end{array}$ \\
\hline Physical layer protocol & PHY 802.11 \\
\hline Packet size & 512 bytes \\
\hline Simulation time & 900 sec \\
\hline
\end{tabular}

\section{RESULT AND DISCUSSION}

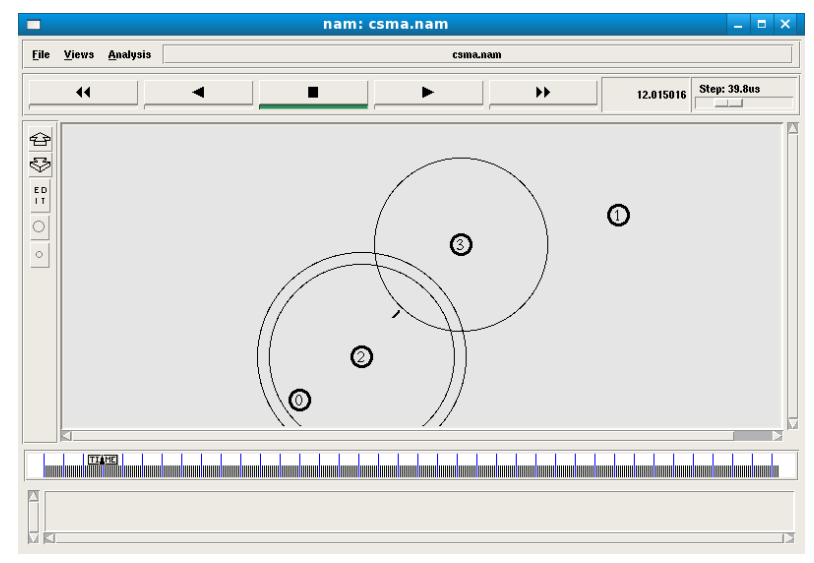

Figure 2 : CSMA Node method

The existing slotted aloha method transmitting the data without propagation. So the data may be dropped. The simulation proceed our proposed CSMA, all the nodes are properly receiving every frames from sender without any failure due to propagated transmission. The graph shows the throughput of both methodologies. The result show, our proposed CSMA reduce packet dropping as well as the outage probability, so the throughput is automatically increased.

The ratio of the OP of unslotted ALOHA and CSMA over that of slotted ALOHA as a function of $\beta$ sens $=\beta r e q=\beta$, for $(M)=,(2,1)$ and a high density of $\lambda=0.2$. For low values of $\beta$, CSMARX yields up to $10 \%$ lower OP compared to unslotted ALOHA, while CSMATX yields $32 \%$ higher OP.

Fig .1. Dataflow Diagram 


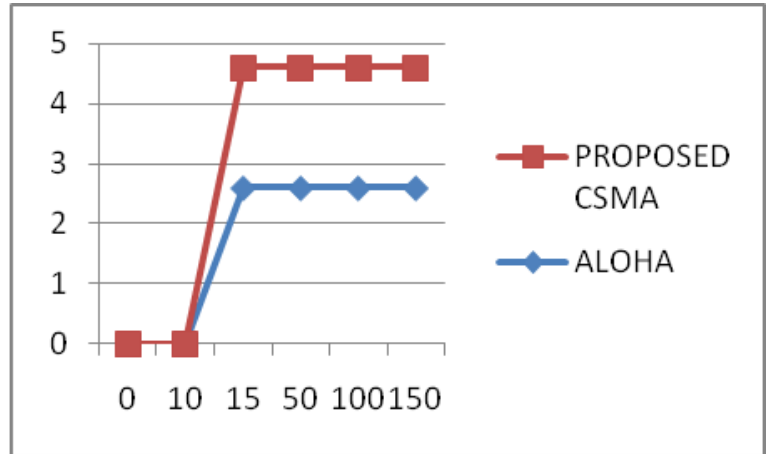

Figure 3 : Graph for both ALOHA and CSMA

However, as $\beta$ increases (i.e., for $\beta>-6 \mathrm{~dB}$ ), making both the sensing zone and the communication zone grow, the OP of CSMATX decreases below that of unslotted ALOHA. This is because the ratio of the area within which the arrival of an interferer causes outage in CSMATX over that in ALOHA (i.e., $(\mathrm{RX} 0, s))$ decreases with $\beta$. More interestingly, for even higher values of $\beta$ (i.e., for $\beta>8 \mathrm{~dB}$ ), both CSMA protocols actually perform better than slotted ALOHA.

\section{CONCLUSION AND FUTURE WORK}

In this paper, the QOS (Quality of service) is increased using on-demand protocol which reduced the energy usage and reduces the cost of transmission according to the individual energy of the node network lifetime will increasing. In future work we can compare our work with enhanced Mac protocol which is having the multipath transmission as their complicity. And which randomly increased the better throughput. The use of multiple channels can certainly increase MAC protocol performance with low energy consumption

\section{REFERENCES}

[1] Mariam Kaynia, NiharJindal,andGeir E. Øien, "Improving the Performance of Wireless Ad Hoc Networks Through MAC Layer Design" IEEE Trans. Wireless Commun., Vol. 10, No. 1, Jan 2011

[2] M. Kaynia and N. Jindal, "Performance of ALOHA and CSMA in spatially distributed wireless networks," in Proc. IEEE International Conf. on Communications (ICC), pp. 1108-1112, Beijing, China, May 2008.

[3] M. Haenggi, "Outage, local throughput, and capacity of random wireless networks," IEEE Trans. Wireless Commun., vol. 8, pp. 4350-4359, Aug. 2009.

[4] N. Jindal, J. Andrews, and S. Weber, "Optimizing the SINR operating point of spatial networks," in Proc. Workshop on Info. Theory and its Applications, San Diego, CA, Jan. 2007.

[5] A. Hasan and J. G. Andrews, "The guard zone in wireless ad hoc networks," IEEE Trans. Wireless Commun., vol. 6, no. 3, pp. 897-906, Dec. 2005.

[6] S. P. Weber, X. Yang, J. G. Andrews, and G. de Veciana, "Transmission capacity of wireless ad hoc networks with outage constraints," IEEE Trans. Inf. Theory, vol. 51, no. 12, pp. 4091-4102, Dec. 2005.

[7] Eun-Sun Jung, Nitin H. Vaidya, A Power Control MAC Protocol for Ad Hoc Networks, Proc. of the International Conference on Mobile Computing and Networking (MOBICOM'02), pp.36-47, Sep, 2002

[8] S. Agarwal, S.Krishnamurthy, R.H.Katz, and S.K.Dao, Distributed Power Control in Ad-hoc Wireless Networks, Proc. of the 12th IEEE International Symposium on Personal, Indoor and Mobile Radio Communications (PIMRC01), 2001 